\title{
Selected Papers of Morikazu Toda
}




\section{SERIES IN PURE MATHEMATICS}

Editor: C C Hsiung

Associate Editors: S S Chern, S Kobayashi, I Satake, Y-T Siu, W-T Wu and M Yamaguti

\section{Part I. Monographs and Textbooks}

Volume 1: Total Mean Curvature and Submanifolds on Finite Type

B Y Chen

Volume 3: Structures on Manifolds

$K$ Yano \& M Kon

Volume 4: Goldbach Conjecture

Wang Yuan (editor)

Volume 6: Metric Rigidity Theorems on Hermitian Locally Symmetric Manifolds Ngaiming Mok

Volume 7: The Geometry of Spherical Space Form Groups

Peter B Gilkey

Volume 9: Complex Analysis

TO Moore \& E H Hadlock

Volume 10: Compact Riemann Surfaces and Algebraic Curves

Kichoon Yang

Volume 13: Introduction to Compact Lie Groups

Howard D Fegan

\section{Part II. Lecture Notes}

Volume 2: A Survey of Trace Forms of Algebraic Number Fields P E Conner \& R Perlis

Volume 5: Measures on Infinite Dimensional Spaces

$Y$ Yamasaki

Volume 8: Class Number Parity

$P E$ Conner \& J Hurrelbrink

Volume 11: Topics in Mathematical Analysis

Th M Rassias (editor)

Volume 12: A Concise Introduction to the Theory of Integration

Daniel W Stroock

\section{Part III. Collected Works}

Volume 14: Selected Papers of Wilhelm P. A. Klingenberg

Volume 15: Collected Papers of $Y$. Matsushima 


\section{Series in Pure Mathematics - Volume 18}

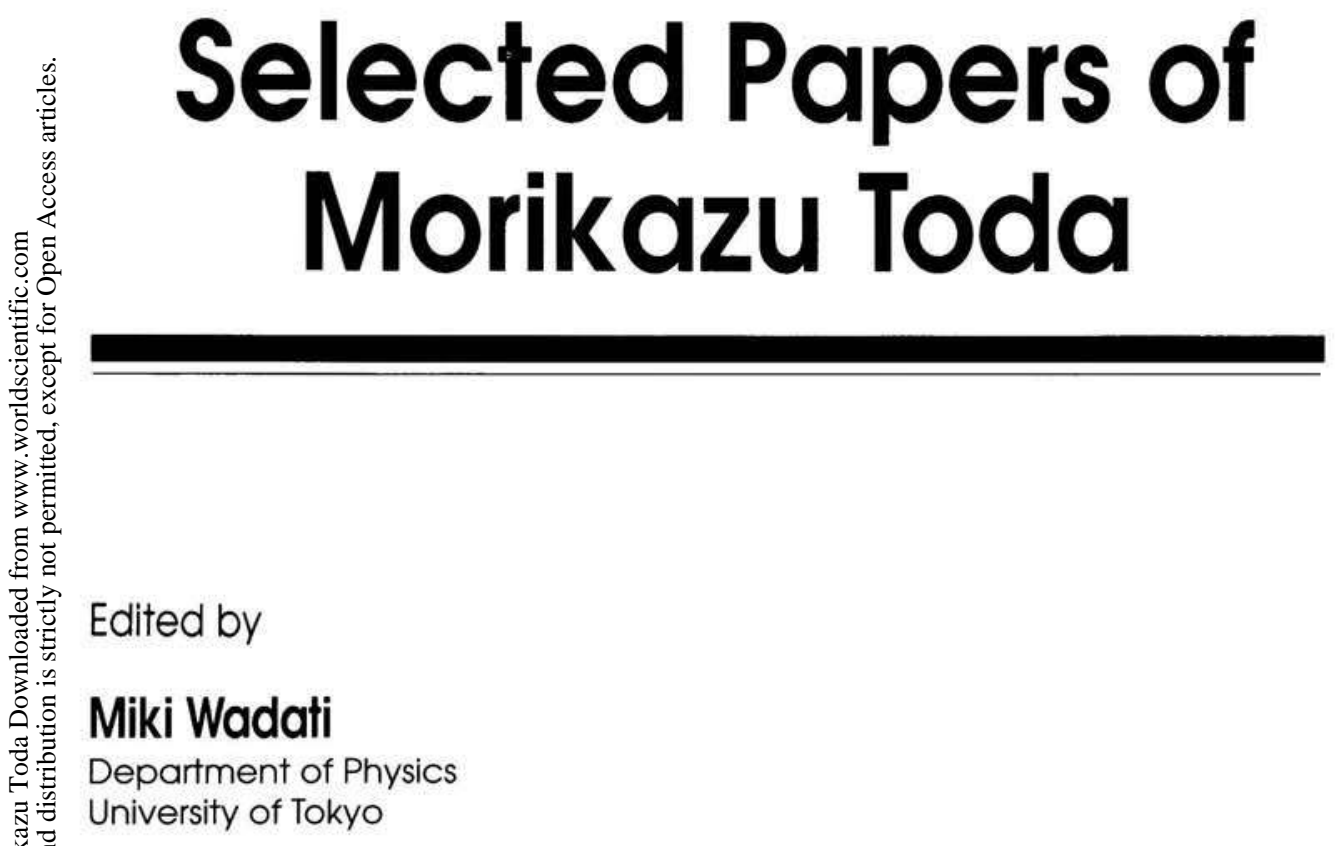




\author{
Published by \\ World Scientific Publishing Co. Pte. Ltd. \\ P O Box 128, Farrer Road, Singapore 9128 \\ USA office: Suite 1B, 1060 Main Street, River Edge, NJ 07661 \\ UK office: 73 Lynton Mead, Totteridge, London N20 8DH
}

\title{
SELECTED PAPERS OF MORIKAZU TODA
}

Copyright $@ 1993$ by World Scientific Publishing Co. Pte. Ltd.

All rights reserved. This book, or parts thereof, may not be reproduced in any form or by any means, electronic or mechanical, including photocopying, recording or any information storage and retrieval system now known or to be invented, without written permission from the Publisher.

For photocopying of material in this volume, please pay a copying fee through the Copyright Clearance Center, Inc., 27 Congress Street, Salem, MA 01970, USA.

ISBN $981-02-1469-3$

Printed in Singapore by JBW Printers \& Binders Pte. Ltd. 


\section{Foreword}

This volume contains selected papers of Dr. Morikazu Toda. The arrangement of papers is in chronological order of publishing dates. His contributions to theoretical physics are legion. There is little need to tell the significance of these papers which speak for themselves. I think, however, it may be interesting and convenient to the reader to mention briefly his scientific career.

Morikazu Toda was born in Tokyo on the 20th of October 1917. He graduated from the Department of Physics, University of Tokyo, in 1940. After serving as an associate professor first at Keijo University and then Tokyo University of Education (Kyoiku University), he was promoted to professor in 1952 at the age of 34 . He was a professor at Chiba University, Yokohama National University and University of the Air. Meanwhile, he was a visiting professor at São Paulo University and University of Trondheim. He is a professor emeritus of Tokyo University of Education and a member of the Royal Norwegian Academy of Science.

Morikazu Toda's main interests are statistical mechanics and condensed matter physics. Among his many contributions, we should mention his works on liquids and nonlinear lattice dynamics. The one-dimensional lattice where nearest neighboring particles interact through an exponential potential is called the Toda lattice. The Toda lattice is a miracle and indeed a jewel in theoretical physics. He received the Mainichi Shuppan-Bunka prize for his contribution to the theory of liquids in 1947 and the Fujiwara prize for discovery of the Toda lattice in 1981.

I am grateful to Professor Akinobu Shimizu and Dr. Noriko Saitoh, Yokohama National University, for their efforts in preparing this work. I am glad to acknowledge the cooperation of World Scientific Publishing and especially of Ms. H. M. Ho who took charge of compiling this volume. 
This page is intentionally left blank 


\section{Preface}

Some of my scientific papers are collected in this book. My scientific career started just before the Second World War, and some of my works of that time were written in Japanese. Among these, a paper on the virial theorem, translated for this issue, presents a method of statistical thermodynamics. For a period of time, I was then interested in the theory of liquids, and low temperature physics. From about 1957, we had a group of physisists who were interested in exact solutions rather than approximate methods, since computer experiments were just revealing that conventional perturbation methods sometimes failed in grasping specific features of phenomena such as the localization of wave functions and vibrational modes around impurities, enhancement of heat flow by nonlinearity of interaction between lattice particles, and so on.

My study of nonlinear system was advanced when I found papers of J. Ford(1961,64), who examined the vibration of lattices with small number of particles and clarified that certain one-dimensional nonlinear lattices marvellously sustain the character of linear modes (his study was initiated by the famous computer experiment by E. Fermi et al., but I could not see it at that time). I thought there would be a lattice model with particular interaction between particles, which admits exact periodic waves, and finally found the integrable nonlinear lattice system.

It is my great pleasure to thank many friends who have shared interest in the lattice problems and nonlinear problems, including E. Teramoto, H. Matsuda, S. Takeno, F. Yonezawa, N. Saito, T. Kotera, Y. Ichikawa, R. Hirota, M. Wadati, J. Satsuma and S. Watanabe.

It was found desirable to have a collection of some of my papers to distribute among seminars in several laboratories, and first I intended only to bind copies and reprints. However, one of my colleagues kindly suggested to make a book, and this is the outcome. Most of my papers on nonlinear lattices are presented. It also includes some of my earlier works which seem to have some originality according to my own dogma and prejudice. It is my sincere hope that this book may give basis for discussion and development for furture study.

It is a pleasant duty to thank M. Wadati for editing and writing the Foreword. I am grateful to the staffs of the Department of Applied 
Mathematics, Faculty of Engineering, Yokohama National University for kind efforts which enabled the publication of this book, especially to A. Shimizu and N. Saitoh. Thanks are also due to the staff of World Scientific Publishing Co. for kind assistance. 


\section{Contents}

Foreword (M. Wadati)

Preface (M. Toda)

Reprinted papers

The Solid States of $\mathrm{H}_{2}$ and $\mathrm{D}_{2}$

Proc. Phys-Math. Soc. Japan 22 (1940) 503-507

Secondary Electron Emission from Pure Metals

Proc. Phys-Math. Soc. Japan 25 (1943) 207

On the Virial Theorem

Recent Problems in Physics (1948) 93-112

Notes on the Theory of High Polymer Solutions (with A. Ishihara)

J. Polymer Science 7 (1951) 277-287

On the Relation between Fermions and Bosons

J. Phys. Soc. Japan 7 (1952) 230

Notes on Fermi and Bose Statistics (with F. Takano)

J. Phys. Soc. Japan 9 (1954) 14-18

On the Theory of Quantum Liquids. I. Surface Tension and Stress

J. Phys. Soc. Japan 10 (1955) 512-517.

Diffusion in Velocity Space and T:`nsport Phenomena

Transport Processes in Statist. Mech. (1958) 148-154

Localized Vibration and Random Walk (with T. Kotera and Y. Kogure) 61 J. Phys. Soc. Japan 17 (1962) 426-433

Statistical Dynamics of Systems of Interacting Oscillators

(with Y. Kogure)

Progr. Theoret. Phys. Suppl. 23 (1962) 157-171

Some Properties of the Pair Distribution Function

J. Phys. Soc. Japan 19 (1964) 1550-1554.

One-Dimensional Dual Transformation

J. Phys. Soc. Japan 20 (1965) 2095 
One-Dimensional Dual Transformation

Progr. Theoret. Phys. Suppl. 36 (1966) 113-119

Vibration of a Chain with Nonlinear Interaction

J. Phys. Soc. Japan 22 (1967) 431-436

Wave Propagation in Anharmonic Lattices

J. Phys. Soc. Japan 23 (1967) 501-506

Mechanics and Statistical Mechanics of Nonlinear Chains

J. Phys. Soc. Japan, Suppl. 26 (1969) 235-237

Waves in Nonlinear Lattice

Progr. Theoret. Phys. Suppl. 45 (1970) 174-200

The Criterion for the Existence of a Gap in the Optical Band of Disordered Mixed Crystal (unpublished, 1970)

Interaction of Solitons with Electromagnetic Waves

Physica Norvegica 5 (1971) 203-207

An Evidence for the Existence of Kirkwood-Alder Transition (with M. Wadati)

J. Phys. Soc. Japan 32 (1972) 1147

The Exact $N$-Soliton Solution of the Korteweg-de Vries

Equation (with $M$. Wadati)

J. Phys. Soc. Japan 32 (1972) 1403-1411

A Soliton and Two Solitons in an Exponential Lattice and Related Equations (with $M$. Wadati)

J. Phys. Soc. Japan 34 (1973) 18-25

Bäcklund Transformation for the Exponential Lattice

(with M. Wadati)

J. Phys. Soc. Japan 39 (1975) 1196-1203

A Canonical Transformation for the Exponential Lattice (with M. Wadati)

J. Phys. Soc. Japan 39 (1975) 1204-1211

Development of the Theory of a Nonlinear Lattice

Progr. Theoret. Phys. Suppl. 59 (1976) 1-35 
Chopping Phenomenon of a Nonlinear System

(with R. Hirota and J. Satsuma)

Progr. Theoret. Phys. Suppl. 59 (1976) 148-161

Problems in Nonlinear Dynamics

Rocky Mountain J. Math. 8 (1978) 197-209

Solitons and Heat Conduction

Physica Scripta 20 (1979) 424-430

Interaction of Soliton with an Impurity in Nonlinear Lattice

(with S. Watanabe)

J. Phys. Soc. Japan 50 (1981) 3436-3442

Experiment on Soliton-Impurity Interaction in Nonlinear Lattice

Using LC Circuit (with $S$. Watanabe)

J. Phys. Soc. Japan 50 (1981) 3443-3450

The Classical Specific Heat of the Exponential Lattice

(with N. Saitoh)

J. Phys. Soc. Japan 52 (1983) 3703-3705

Interest in Form in Japan and the West

Science on Form (1986) 1-8

Coupled Nonlinear Waves

Physica D 33 (1988) 317-322

Nonlinear Dual Lattice (with Y. Okada and S. Watanabe)

J. Phys. Soc. Japan 59 (1990) 4279-4285

Partition Function of Nonlinear Lattice

Nonlinear Dispersive Wave Systems (1992) 435-443

Academic Career of Morikazu TODA

Bibliography of Morikazu TODA 